\title{
Inhibition of calcineurin/NFATc4 signaling attenuates ventilator-induced lung injury
}

\author{
MIN LI $^{1 *}$, XIANG-ZHI FANG ${ }^{2 *}$, XIAO-TIAN LIU ${ }^{1}$, YONG-FENG ZHENG ${ }^{1}$, \\ YUN-BIN XIE ${ }^{1}$, XIAO-DONG MA ${ }^{1}$, YAN XIA ${ }^{1}$ and DONG-HUA SHAO ${ }^{1}$ \\ ${ }^{1}$ Department of Anesthesiology, Affiliated People's Hospital of Jiangsu University, Zhenjiang, \\ Jiangsu 212002; ${ }^{2}$ Department of Anesthesiology, Clinical Medical School of Yangzhou University, \\ Subei People's Hospital of Jiangsu Province, Yangzhou, Jiangsu 225001, P.R. China
}

Received December 24, 2018; Accepted October 10, 2019

DOI: $10.3892 / \mathrm{mmr} .2019 .10851$

\begin{abstract}
Ventilator-induced lung injury (VILI) is a life-threatening condition caused by the inappropriate use of mechanical ventilation (MV). However, the precise molecular mechanism inducing the development of VILI remains to be elucidated. In the present study, it was revealed that the calcineurin/NFATc4 signaling pathway mediates the expression of adhesion molecules and proinflammatory cytokines essential for the development of VILI. The present results revealed that a high tidal volume ventilation (HV) caused lung inflammation and edema in the alveolar walls and the infiltration of inflammatory cells. The calcineurin activity and protein expression in the lungs were increased in animals with VILI, and NFATc4 translocated into the nucleus following calcineurin activation. Furthermore, the translocation of NFATc4 and lung injury were prevented by a calcineurin inhibitor (CsA). Thus, the present results highlighted the critical role of the calcineurin/NFATc4 signaling pathway in VILI and suggest that this pathway coincides with the release of ICAM-1, VCAM-1, TNF- $\alpha$ and IL-1 $\beta$.
\end{abstract}

\section{Introduction}

Mechanical ventilation (MV) is an important therapy used to assist critically ill patients with moderate or severe ARDS. However, mechanical ventilation can induce or aggravate lung injury, which is referred to as ventilator-induced lung injury (VILI) (1). Increased infiltration of inflammatory cells, alveolar edema, and barrier dysfunction due to excessive mechanical stresses generated during mechanical ventilation

Correspondence to: Dr Dong-Hua Shao, Department of Anesthesiology, Affiliated People's Hospital of Jiangsu University, 8 Dianli Road, Zhenjiang, Jiangsu 212002, P.R. China

E-mail: 13805281211@163.com

${ }^{*}$ Contributed equally

Key words: mechanical ventilation, lung injury, inflammation, $\mathrm{Ca}^{2+}$ have been recognized as the major pathophysiologic events of VILI (2). A growing body of evidence suggests that VILI is essentially an excessive, uncontrolled inflammatory response in the lung $(3,4)$. Despite the existence of lung protective ventilator strategies for improving the ventilation procedures, the morbidity and mortality of VILI have remained high (5). Thus, the investigation of the precise molecular mechanism of VILI is urgently required.

Although the mechanism responsible for initiating VILI is not completely understood, it is believed that inflammatory responses are key factors $(6)$. Studies $(7,8)$ have revealed that disturbance in intracellular ion homeostasis is a major event eliciting both the inflammatory responses and production of inflammatory mediators, such as IL-1 $\beta$ and TNF- $\alpha$. Some studies $(9,10)$ have suggested that a $\mathrm{Ca}^{2+}$ influx mediates the augmentation of many different inflammation and autoimmune diseases. Notably, $\mathrm{Ca}^{2+}$ influx has been proposed to perform an essential function in the development of VILI (11); in contrast, the inhibition of cation channels protects against VILI (12). These studies imply that $\mathrm{Ca}^{2+}$ mobilization plays a critical role in the progression of VILI.

Calcineurin is a calcium-activated protein phosphatase and an essential nodal factor in the regulation of cellular functions (13). An increase in the levels of intracellular $\mathrm{Ca}^{2+}$ activates calcineurin. Activated calcineurin dephosphorylates the nuclear factor of activated T cells (NFAT), which in turn modulates the transcription of target genes (14). In recent years, various inflammatory cytokines and adhesion molecules have been identified as NFAT regulatory targets (15). These molecules include VCAM-1, ICAM-1, IL-6, IL-8 and MCP-1. The inhibition of NFAT was revealed to be an effective method for reducing the multiple inflammatory cytokines induced by TNF- $\alpha$ in human retinal microvascular endothelial cells (16), thus further highlighting NFAT signaling as a potential anti-inflammatory target. Recent studies $(17,18)$ have revealed that NFATc3 is a key molecular regulator of sepsis-induced lung injury. Notably, NFATc4 has been suggested to be a pivotal regulatory event in endothelial cell inflammation (15), which is an early step in the development of lung injury. Therefore, the calcineurin/NFATc4 signaling pathway has also received considerable attention due to its fundamental role in mediating lung injuries. 
However, whether calcineurin/NFATc4 signaling is involved in the development of VILI remains unknown. Thus, it was determined whether the calcineurin/NFATc4 signaling pathway contributes to VILI.

\section{Materials and methods}

Animals. Adult male Wistar rats (weighing 200-230 g) were obtained from the Comparative Medicine Center of YangZhou University. All rats were housed in air-filtered, temperature-controlled units with access to chow and water. All experimental operations were performed with approval by and in accordance with the guidelines set by the Animal Ethics Committee of YangZhou University. The rats were anesthetized by intraperitoneal injection with ketamine hydrochloride $(50 \mathrm{mg} / \mathrm{kg})$ and xylazine $(10 \mathrm{mg} / \mathrm{kg})$.

In the first series of experiments, the rats were randomly divided into the following three groups: The nonventilated control group (Control, $\mathrm{n}=8$ ); the ventilated with a low tidal volume ( $6 \mathrm{ml} / \mathrm{kg}$ ventilation) for 6 -h group $(\mathrm{LV}, \mathrm{n}=8)$; and the ventilated with a high tidal volume (30 ml $/ \mathrm{kg}$ ventilation) for 6-h group $(\mathrm{HV}, \mathrm{n}=8)$.

In the second series of experiments, the rats were randomly divided into the following four groups: the nonventilated control group (Control, $\mathrm{n}=8$ ); the pretreated with cyclosporine A group $(\mathrm{CsA}, \mathrm{n}=8)$; the ventilated with a high tidal volume $(30 \mathrm{ml} / \mathrm{kg}$ ventilation) for 6-h group ( $\mathrm{HV}, \mathrm{n}=8)$; and the $\mathrm{HV}$-treated with cyclosporine A group ( $\mathrm{HV}+$ cyclosporine $\mathrm{A}, \mathrm{n}=8$ ).

The CsA-treated rats were administered cyclosporine A (Abcam) $(7 \mathrm{mg} / \mathrm{kg}$ ), which is a selective inhibitor of calcineurin, by intraperitoneal injection. The HV + cyclosporine A-treated rats were intraperitoneally administered/pretreated with cyclosporine A $(10 \mathrm{mg} / \mathrm{kg}) 1 \mathrm{~h}$ before MV. Upon completion of the experiments, rats were anesthetized with i.p. ketamine hydrochloride $(50 \mathrm{mg} / \mathrm{kg})$ and xylazine $(10 \mathrm{mg} / \mathrm{kg})$ and sacrificed by exsanguinations from their inferior vena cava.

Induction of VILI. The rats underwent tracheotomies and were intubated with an $18-\mathrm{G}$ angiocatheter. Then, the rats were connected to a volume-limited rodent ventilator (DW3000; Zhenghua Biologic Apparatus Facilities Co.,Ltd.). The rats were mechanically ventilated for $6 \mathrm{~h}$ using either a low tidal volume (VT=6 ml/kg) or a high tidal volume (VT=30 ml/ $\mathrm{kg}$ ) with a positive end-expiratory pressure of $0 \mathrm{~cm} \mathrm{H}_{2} \mathrm{O}$, a respiratory rate of 40 breaths/min and an $\mathrm{FIO}_{2}$ of $21 \%$ (Fig. 1).

Assessment of lung histology. The lung tissues were fixed and stained with hematoxylin and eosin (H\&E) as previously described (5). Two investigators blinded to group assignments analyzed the samples and determined levels of lung injury according to the semiquantitative scoring outlined below. All lung fields at an x20 magnification were examined for each sample. Assessment of histological lung injury was performed according to combined assessments of alveolar septal thickening, inflammatory infiltration, microatelectasis or alveolar overdistension, and microhemorrhage. Neutrophils were counted in the alveolar airspaces, alveolar walls, and interstitium. The injury scores were as follows: 0 , absent with normal appearance; 1 , slight injury ( $<30 \%$ lung section); 2 , intermediate injury (30-60\% lung section); and 3 , severe injury ( $>60 \%$ lung section).

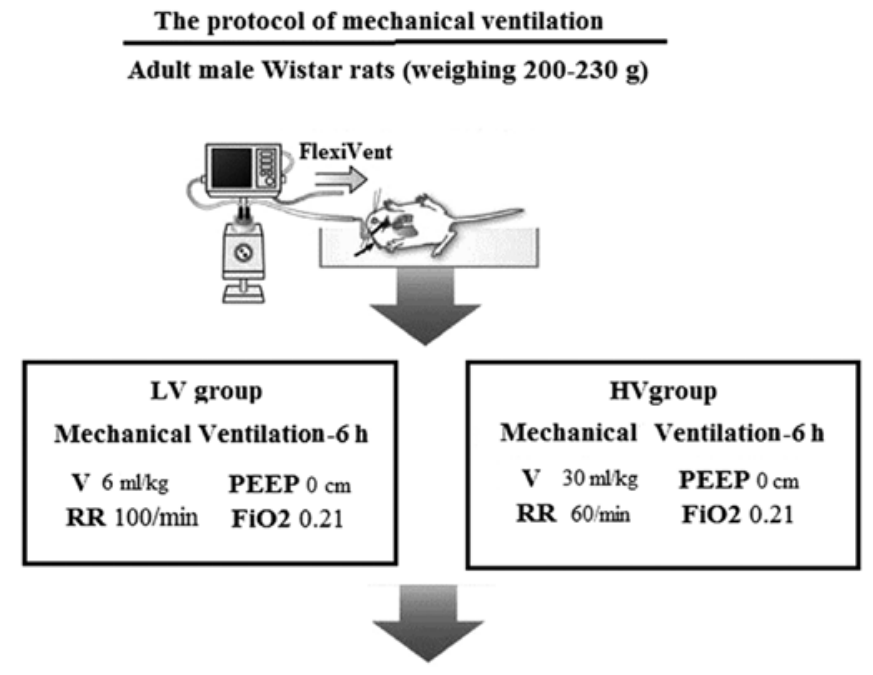

Sacrifice, BAL and harvest lung tissue

Figure 1. The protocol of mechanical ventilation lung injury model. LV, ventilated with a low tidal volume; HV, ventilated with a high tidal volume; BAL; bronchoalveolar lavage.

Measurement of protein concentrations. The bronchoalveolar lavage fluid (BALF) was harvested by injecting and retrieving sterile PBS three times through the tracheal intubation. The BALF $(4 \mathrm{ml})$ was centrifuged at $4^{\circ} \mathrm{C}$ and $300 \mathrm{x}$ g for $10 \mathrm{~min}$. The total protein concentrations in the BALF were determined using a bicinchoninic acid (BCA) protein assay kit (Beyotime Institute of Biotechnology) according to the manufacturer's instructions.

Wet-to-dry lung weight ratio. The right upper lobe of the lungs was weighed to measure the wet weight. The dry weight was determined after heating the lungs at $80^{\circ} \mathrm{C}$ for $48 \mathrm{~h}$.

Myeloperoxidase (MPO) activity bioassay. MPO can be used as an index of lung neutrophil infiltration. The MPO activity in the lung tissues was assayed by using an MPO assay kit (Jiancheng Bioengineering Institute of Nanjing). Briefly, the lung tissues were homogenized and centrifuged at $12,000 \mathrm{xg}$ and $4^{\circ} \mathrm{C}$ for $10 \mathrm{~min}$. An MPO activity assay was performed at $450 \mathrm{~nm}$ to calculate the MPO activity.

RT-PCR. The total RNA in the lung tissue was harvested using TRIzol reagent (Invitrogen; Thermo Fisher Scientific, Inc.). cDNA was synthesized using qRT SuperMix (Vazyme, JiangSu, China). RT-qPCR was performed with AceQ SYBR-Green Master Mix (Vazyme). The following primers were used for PCR analysis: ICAM-1 forward, 5'-GTGATG CTCAGGTATCCATCCA-3' and reverse, 5'-CACAGTTCT CAAAGCACAGCG-3'; VCAM-1 forward, 5'-TTGGGAGCC TCAACGGTACT-3' and reverse, 5'-GCAATCGTTTTGTAT TCAGGGGA-3'; TNF- $\alpha$ forward, 5'-GGAACACGTCGT GGGATAATG-3' and reverse, 5'-GGCAGACTTTGGATG CTTCTT-3'; IL-8 forward, 5'-AGCAGTCCCAACTAAGCA GTA-3' and reverse, 5'-CAGCCAGTAGAGGATGCTGA-3'; and $\beta$-actin forward, 5'-GAGTCCTACGACATCATCGCT-3' and reverse, 5'-CGTCCGACATAGTTTGGGAAA-3'. The 
A

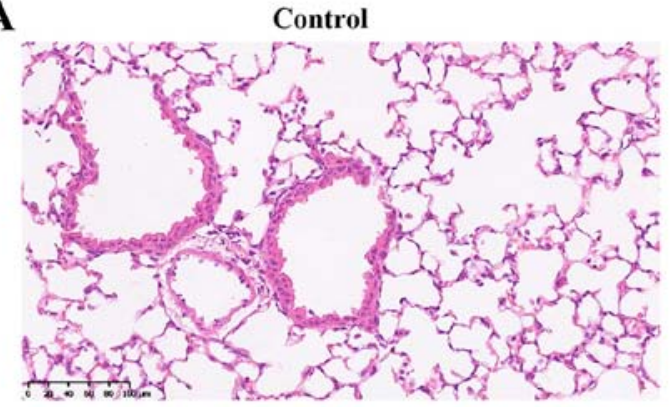

C

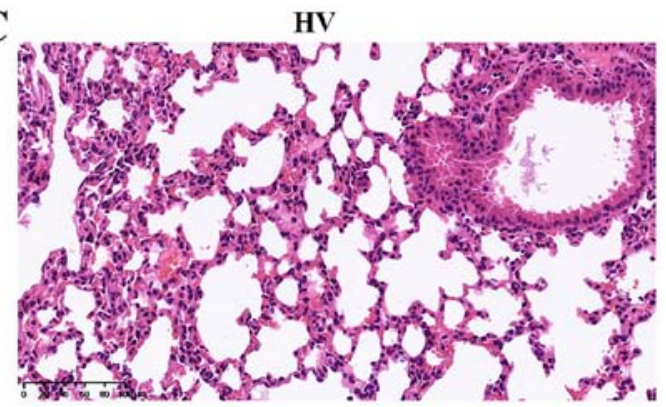

B

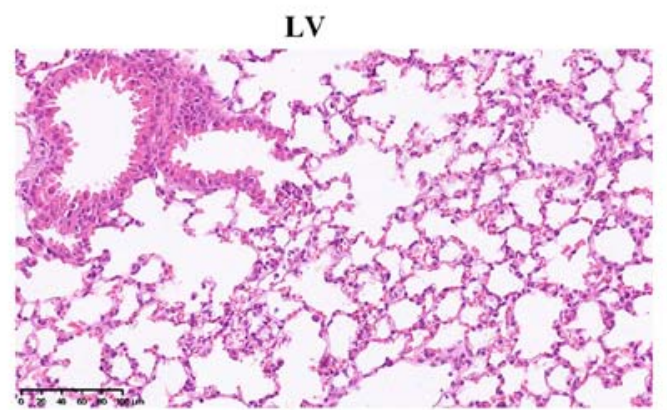

D

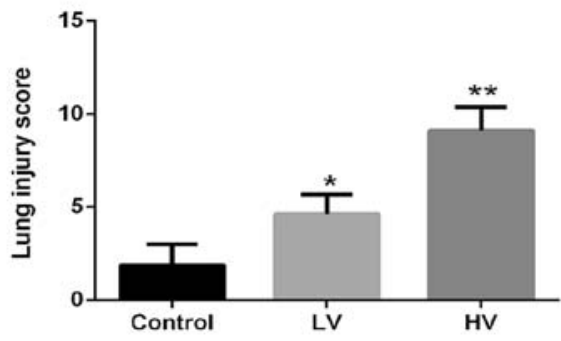

Figure 2. MV induces pathological changes in the lungs of rats. After the rats were treated with $\mathrm{MV}(6 \mathrm{ml} / \mathrm{kg} \mathrm{or} 20 \mathrm{ml} / \mathrm{kg})$, the following results were observed: Histopathological changes (x200, magnification) in the lungs of (A) control, (B) LV and (C) HV groups. (D) Histological injury scores of the lungs. The data are presented as the means $\pm \mathrm{SD}(\mathrm{n}=8) .{ }^{*} \mathrm{P}<0.05,{ }^{* *} \mathrm{P}<0.01$, compared with the Control group. MV, mechanical ventilation; $\mathrm{LV}$, ventilated with a low tidal volume; $\mathrm{HV}$, ventilated with a high tidal volume.

cycle threshold (CT) method was used to analyze the data in each experimental group (19).

Calcineurin activity analysis. The lung tissues of the rats were weighed and homogenized in RIPA lysis buffer (P0013B, Beyotime Institute of Biotechnology) for $30 \mathrm{~min}$; then, the total protein levels were measured using a BCA assay. The protein lysates were used immediately for the CaN activity assay; specifically, the Calcineurin Activity Assay Kit (Nanjing Jiancheng Bioengineering Institute) was used.

Preparation of cytoplasmic and nuclear extracts. The isolation of the cytoplasmic and nuclear extracts was performed using a cytoplasmic and nuclear protein extraction kit (cat. no. R0050; Solarbio Life Sciences). Briefly, $50 \mathrm{mg}$ lung tissues were weighed, homogenized and centrifuged in ice-cold PBS at $500 \mathrm{x} \mathrm{g}$ for $3 \mathrm{~min}$. Then, the sediments were dissolved in $200 \mu 1$ of cytoplasmic extraction reagent by vortexing, and centrifuged at $12,000 \mathrm{x} \mathrm{g}$ for $10 \mathrm{~min}$ at $4^{\circ} \mathrm{C}$. The supernatant was harvested as the cytoplasmic fraction. The sediments were re-dissolved in $100 \mu \mathrm{l}$ of nuclear extraction buffer by vortexing for $15 \mathrm{sec}$ and incubated on ice for $10 \mathrm{~min}$, and then centrifuged for $10 \mathrm{~min}$ at $16,000 \mathrm{xg}$ at $4^{\circ} \mathrm{C}$. Then, the supernatant was harvested as the nuclear fraction. The concentrations of each extract were assessed using a BCA protein assay kit (Beyotime Institute of Biotechnology).

Western blotting. Western blotting was performed as previously described (20). Equal amounts $(20 \mu \mathrm{l})$ of sample buffer were loaded onto a 10\% SDS-PAGE gel. The protein was electrotransferred to polyvinylidene difluoride (PVDF) membranes. Then, the membranes were incubated with the following primary antibodies at $4^{\circ} \mathrm{C}$ for $12 \mathrm{~h}$ : Anti-inhibitors against
NFATc4 (ab99431, 1:1,000; Abcam), VCAM-1 (ab134047, 1:1,000; Abcam), ICAM-1 (10020-1-AP, 1:1,000; Proteintech), IL-8 (ab7747, 1:1,000; Abcam), TNF- $\alpha$ (17590-1-AP, 1:1,000; Proteintech), Lamin B (AF1408, 1:1,000; Beyotime Institute of Biotechnology) and $\beta$-actin (20536-1-AP 1:1,000; Proteintech). The membranes were incubated at room temperature for $1 \mathrm{~h}$ with a peroxidase-conjugated secondary antibody $(1: 2,000$; Beyotime Institute of Biotechnology). The protein bands were visualized using a ChemiDoc imaging system (Bio-Rad Laboratories, Inc.), and the intensity was quantified using ImageJ 1.8.0 (National Institutes of Health).

Statistical analysis. The data are presented as the mean \pm standard deviation (SD). The statistical analysis was performed via one-way analysis of variance with Bonferroni multiple comparison tests using GraphPad Prism V.5.01 (GraphPad, Inc.). The data were considered to be statistically significant when the P-value was $<0.05$.

\section{Results}

MV induces lung injury and inflammatory responses. A histologic examination was performed to evaluate the lung tissue damage induced by MV. Consistent with previous studies, the results revealed severe lung injury characterized by neutrophil accumulation and edema disruption in the lung tissues in the $\mathrm{HV}$ group. The histological injury score in the HV group was markedly higher than that in the control group $(\mathrm{P}<0.01)$ (Fig. 2A-D).

To further clarify the effect of MV in lung injury, the changes in the wet/dry ratios and the total protein concentrations were investigated. The results demonstrated that MV induced a significant increase in the wet/dry ratios $(\mathrm{P}<0.01)$ 

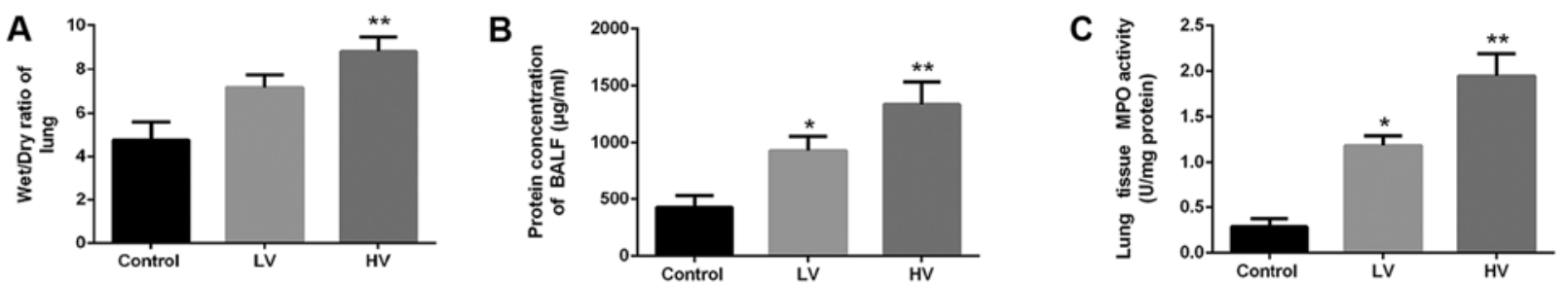

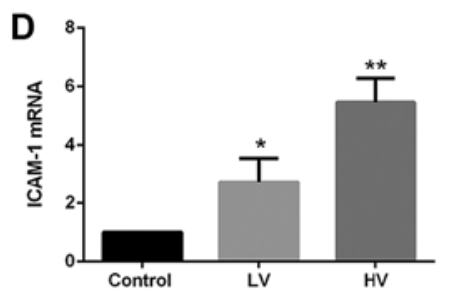

E

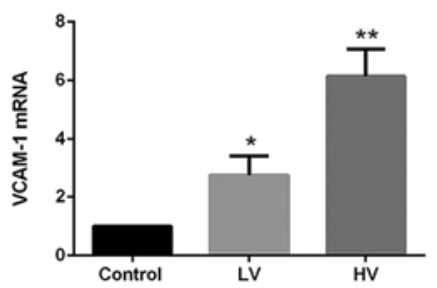

H

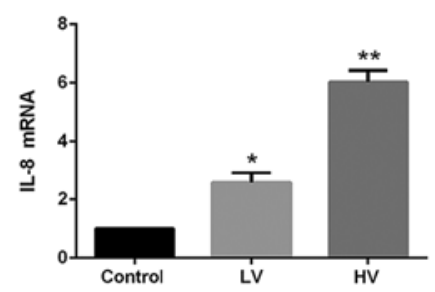

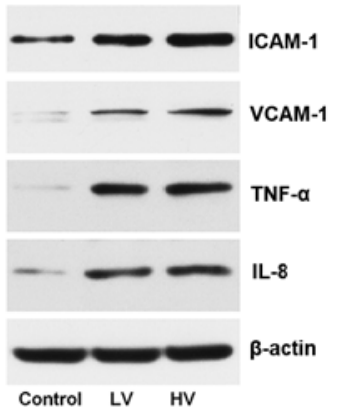

$\mathbf{F}$
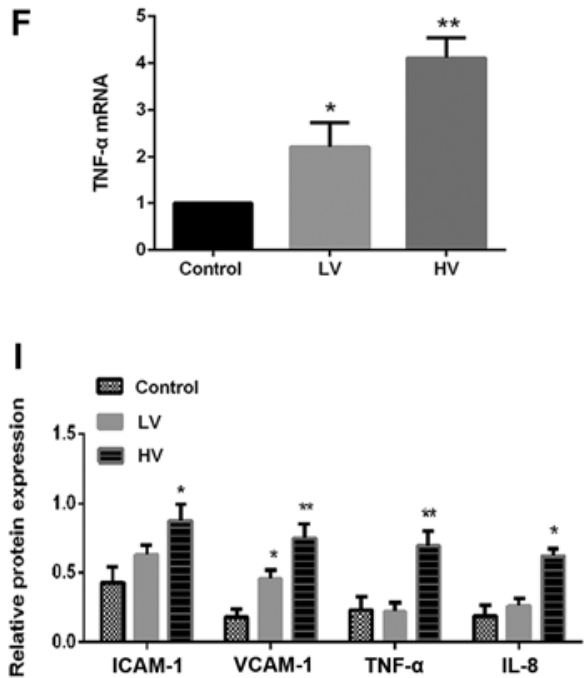

Figure 3. MV induces edema and an inflammatory response in the lungs of rats. After the rats were treated with MV (6 ml $/ \mathrm{kg} \mathrm{or} 20 \mathrm{ml} / \mathrm{kg}$ ), pulmonary edema was determined by (A) the wet/dry weight ratio and (B) the protein levels in BALF. (C) Lung MPO activity was also assessed. The mRNA levels of (D) ICAM-1, (E) VCAM-1, (F) TNF- $\alpha$ and (G) IL-8 were assessed. (H) The protein expression of VCAM-1, ICAM-1, TNF- $\alpha$ and IL-8 were assessed. Blots are representative of at least three independent experiments. (I) Semi-quantification of immunoblots shown in (H). The data are presented as the means $\pm \mathrm{SD}(\mathrm{n}=8)$. " $\mathrm{P}<0.05$, ${ }^{* *} \mathrm{P}<0.01$, compared with the Control group. MV, mechanical ventilation; LV, ventilated with a low tidal volume; HV, ventilated with a high tidal volume.

(Fig. 3A) and total protein concentrations of BALF $(\mathrm{P}<0.01)$ (Fig. 3B).

To further confirm the effects of MV in the inflammatory reactions, the expression levels of adhesion molecules and proinflammatory cytokines and MPO activity were examined. Significant regulation of ICAM-1, VCAM-1, TNF- $\alpha$ and IL-8 in the HV group rats was observed $(\mathrm{P}<0.01)$ (Fig. 3D-I). Similarly, it was revealed that MV promoted neutrophil infiltration into the lung tissues, which was reflected by the increased expression of lung MPO activity (Fig. 3C). These results provided indirect evidence of the involvement of the inflammatory response.

MV activates the calcineurin/NFAT4 signaling pathway in the lungs. Calcineurin is a calcium-activated protein phosphatase. Activated calcineurin dephosphorylates NFATs, which then translocate to the cell nucleus to regulate the transcription of NFAT-responsive genes. To investigate whether the calcineurin/NFATc4 pathway influences VILI, the activation of the calcineurin/NFATc4 signaling pathway was examined. Fig. 4A and $\mathrm{B}$ demonstrated that the activity and protein expression of $\mathrm{CaN}$ were significantly increased after MV $(\mathrm{P}<0.01)$

Subsequently, the effects of MV on the phosphorylation and distribution of NFATc 4 were examined. As revealed in Fig. 4C and D, the levels of the nuclear translocation of NFATc 4 were increased, and the levels of the cytoplasmic phosphorylation and translocation of NFATc4 were also decreased. These results indicate that MV exposure could activate lung calcineurin/NFAT4 signaling.

VILI is suppressed by calcineurin/NFATc4 signaling antagonists. To further confirm the role of calcineurin/NFATc4 pathway activation in VILI, lung injury after VILI in the presence of calcineurin antagonists was examined. The treatment with the calcineurin antagonists significantly reduced the VILI pathological changes $(\mathrm{P}<0.05)$, the wet/dry ratios $(\mathrm{P}<0.05)$, the total protein concentrations in BALF and MPO activity $(\mathrm{P}<0.05)$ (Fig. 5A-E). In contrast, the lung injury in the naive rats was not affected by the treatment with CsA), indicating that CsA improved VILI but did not affect normal lung tissue. These results indicated that the calcineurin/NFATc4 pathway plays an essential role in VILI.

Inhibition of calcineurin/NFATc4 signaling suppresses the inflammatory response of $M V$. Since inflammatory responses play an important role in VILI, whether the administration of calcineurin inhibitors could reverse the increase in the expression levels of adhesion molecules and proinflammatory cytokines was investigated. The results demonstrated that the 
A

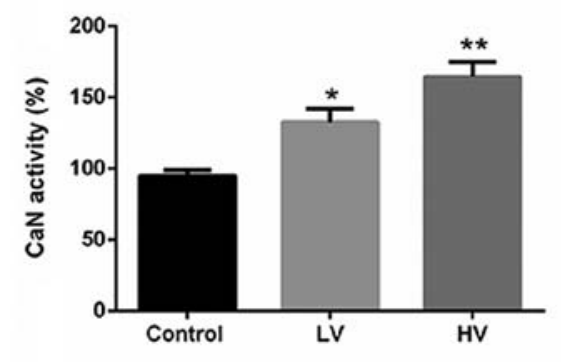

C

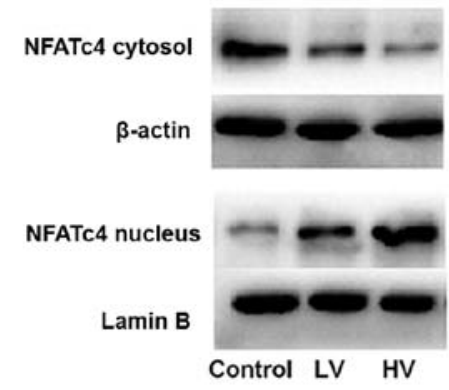

B

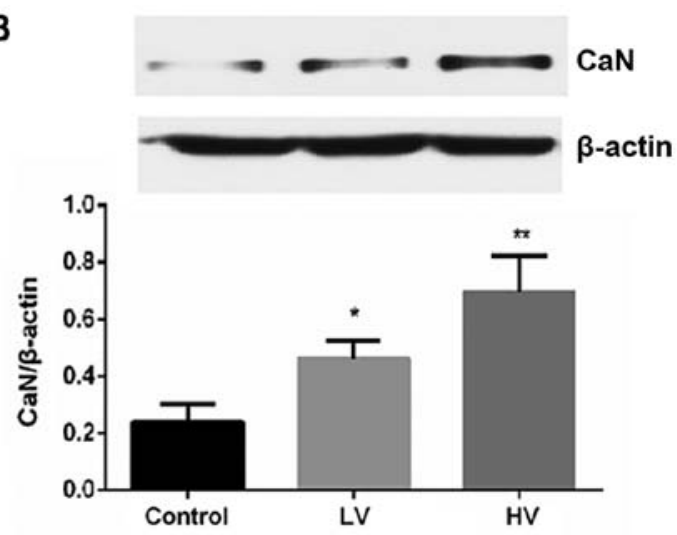

D

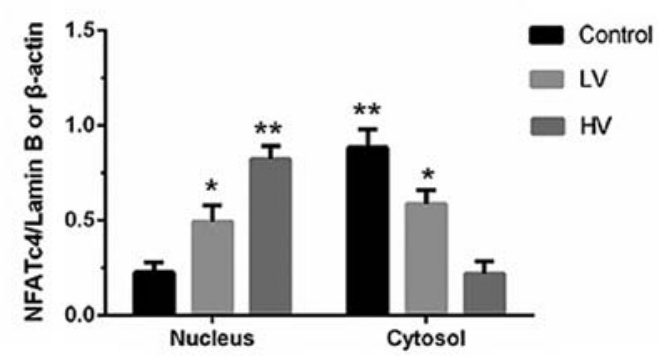

Figure 4. MV activates the calcineurin/NFAT4 signaling pathway in the lungs. (A) CaN activity and (B) protein expression were significantly increased after MV. (C and D) The protein levels of NFATc4 in the cytoplasmic and nuclear fractions were determined by immunoblotting. Lamin B and $\beta$-actin served as loading controls for the nuclear and cytoplasmic fractions, respectively. The data are presented as the means $\pm \mathrm{SD}(\mathrm{n}=8)$. ${ }^{*} \mathrm{P}<0.05$, ${ }^{* *} \mathrm{P}<0.01$, compared with the Control group. MV, mechanical ventilation; $\mathrm{CaN}$, calcineurin; $\mathrm{LV}$, ventilated with a low tidal volume; HV, ventilated with a high tidal volume.

A

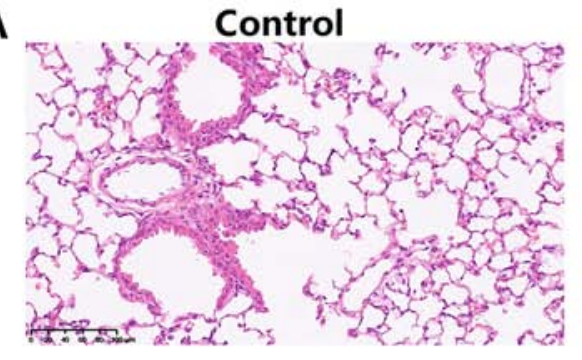

CsA

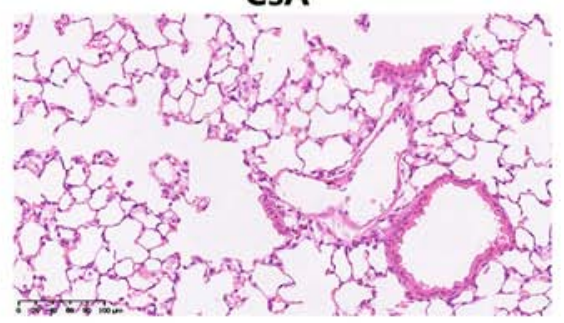

HV

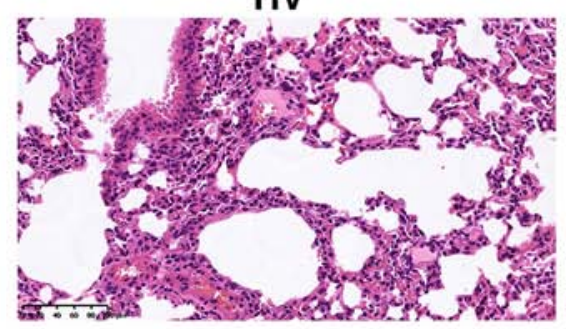

CsA+HV

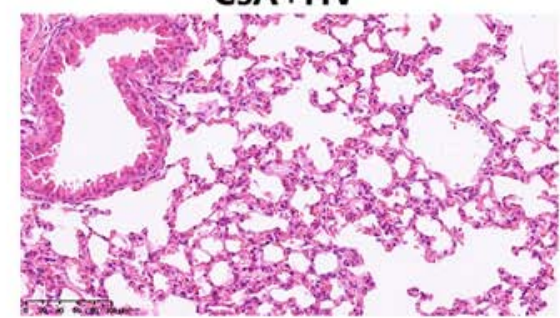

B

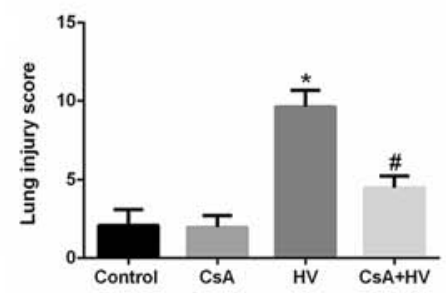

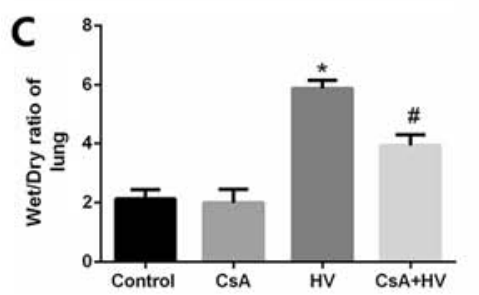

D

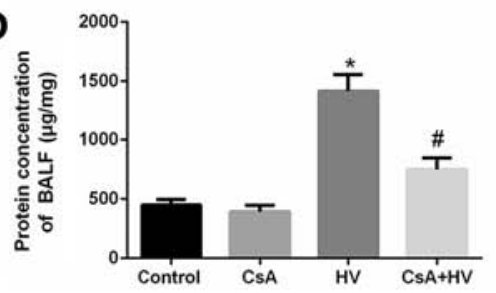

$\mathbf{E}$

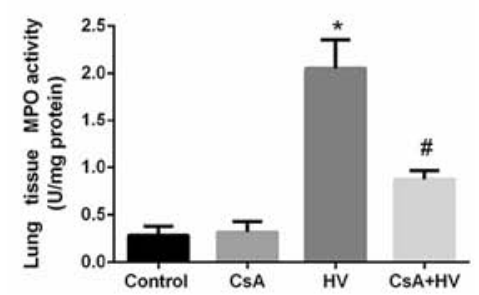

Figure 5. Disruption of the calcineurin/NFAT4 pathway attenuates MV-induced pathological changes, edema and MPO activity in the lungs of rats. (A and B) Pretreatment with CsA inhibited MV-induced pathological changes. Pretreatment with CsA decreased (C) the wet/dry weight ratios and (D) the protein levels of BALF. (E) Pretreatment with CsA inhibited the MV-induced MPO activity. The data are presented as the means \pm SD ( $n=8$ ). ${ }^{*} \mathrm{P}<0.05$ compared with the Control group; ${ }^{\prime} \mathrm{P}<0.05$ compared with the HV group. $\mathrm{MV}$, mechanical ventilation; CsA, calcineurin inhibitor; $\mathrm{LV}$, ventilated with a low tidal volume; $\mathrm{HV}$, ventilated with a high tidal volume. 
A
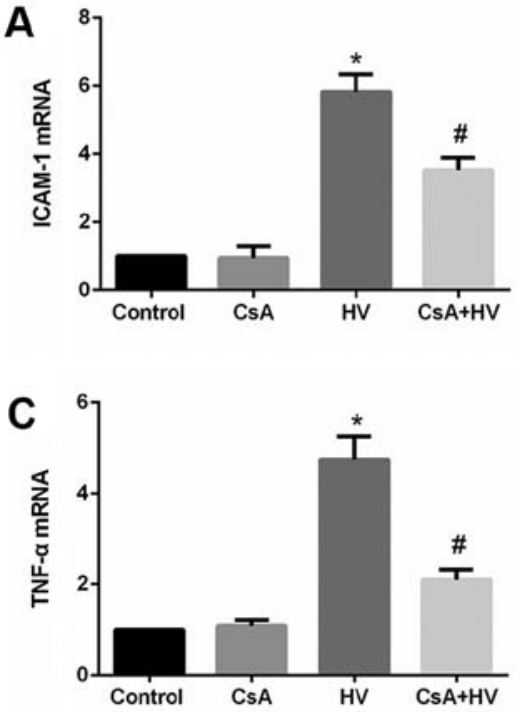

E

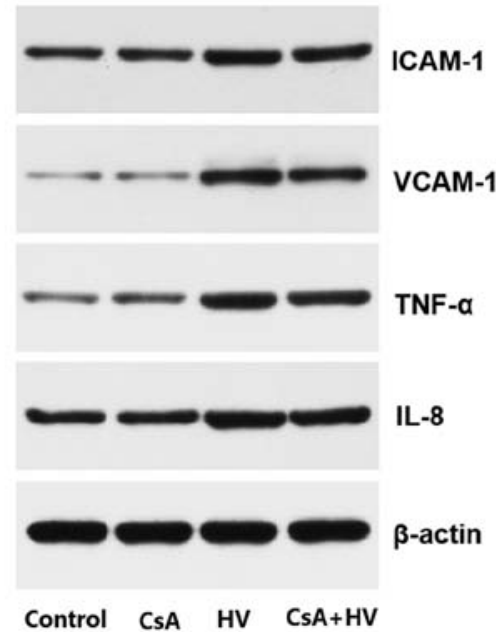

B

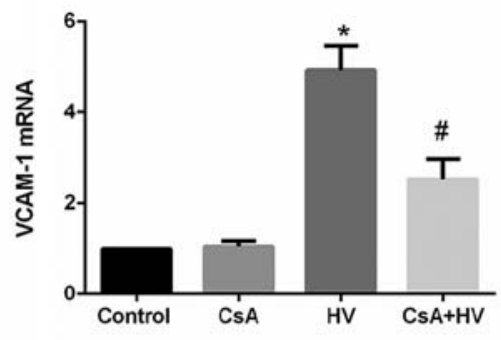

D

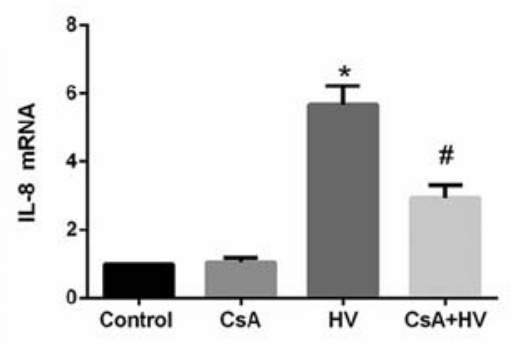

$\mathbf{F}$

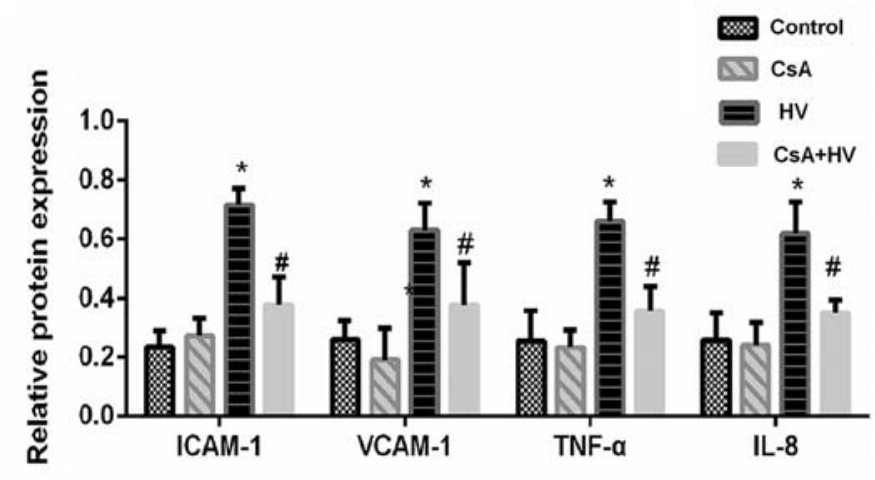

Figure 6. Proinflammatory cytokine and adhesion molecule production induced by MV depends on calcineurin/NFAT4 signaling. Pretreatment with CsA decreased the mRNA levels of (A) ICAM-1, (B) VCAM-1, (C) TNF- $\alpha$ and (D) IL-8. (E and F) Pretreatment with CsA decreased the protein expression of ICAM-1, VCAM-1, TNF- $\alpha$ and IL-8. The data are presented as the means \pm SD $(n=8) .{ }^{*} \mathrm{P}<0.05$ compared with the Control group; ${ }^{*} \mathrm{P}<0.05$ compared with the HV group. MV, mechanical ventilation; CsA, calcineurin inhibitor; LV, ventilated with a low tidal volume; HV, ventilated with a high tidal volume.

pretreatment with CsA prevented the increases in ICAM-1, VCAM-1, TNF- $\alpha$ and IL-8 levels $(\mathrm{P}<0.05)$ (Fig. 6A-F). These results further indicated that the calcineurin/NFATc4 pathway may be involved in the inflammatory responses of VILI.

\section{Discussion}

The present study revealed that calcineurin/NFATc4 signaling plays a critical role in the development of VILI. The principle findings are as follows: i) MV caused the activation of the calcineurin/NFATc4 pathway, which was associated with the degree of lung injury in the rats; ii) calcineurin/NFATc4 signaling activation in the lungs led to increased levels of adhesion molecules and proinflammatory cytokines; iii) the inhibition of the calcineurin/NFATc4 signaling pathway attenuated VILI and the release of pro-inflammatory cytokines. Collectively, the present results highlighted the critical role of the calcineurin/NFATc4 signaling pathway in VILI.
Although essential in critically ill patients, mechanical ventilation often induces or aggravates lung injury, which is termed VILI $(21)$. Accumulating studies $(22,23)$ have revealed that VILI is mainly characterized by increases in pulmonary vascular permeability, influxes of inflammatory cells and various cytokines. These inflammatory cells could produce inflammatory cytokines. Furthermore, these cytokines could facilitate the infiltration of inflammatory cells (24). Therefore, the inflammatory response is critical for the development of VILI. However, the mechanisms of VILI remain unclear. In the present study, it was demonstrated that the calcineurin/NFAT4 signaling pathway was activated during VILI. A high-VT MV for $6 \mathrm{~h}$ activated both calcineurin and NFATc4; conversely, the blockage of the calcineurin/NFATc4 signaling pathway with cyclosporine A attenuated VILI. These findings provide strong evidence that the calcineurin/NFATc4 signaling pathway may be among the most important signaling pathways activated by MV and, thus, is involved in the development of VILI. 
Calcineurin is a calcium-activated protein phosphatase that has been implicated in various inflammatory diseases (25). Activated calcineurin dephosphorylates the nuclear factor of activated T cells (NFAT), which, in turn, modulates the transcription of target genes associated with cellular proliferation, differentiation, inflammation and angiogenesis (14). The NFAT protein family consists of the following five members: NFATc1, NFATc2, NFATc3, NFATc4 and NFAT5 [tonicity-responsive enhancer-binding protein (TonEBP)] (26). NFATc3 has been revealed to play an important role in the activation of macrophages during sepsis $(17,18)$. Collectively, these experimental studies indicated that the calcineurin/NFATc 4 signaling pathway plays a fundamental role in mediating inflammation in lung injury. The present study extended the understanding of the important role of calcineurin/NFATc4 signaling in lung injury by demonstrating that the calcineurin/NFATc4 pathway contributes to VILI.

In the present study, it was observed that MV induced the calcineurin/NFATc4-dependent production of adhesion molecules and pro-inflammatory cytokines. These adhesion molecules and proinflammatory cytokines are known to serve as an important regulatory event to deteriorate VILI (27). On the basis of the present findings, it was speculated that the mechanical stresses generated during mechanical ventilation can activate the calcineurin/NFATc4 signaling pathway, resulting in increases in adhesion molecules and proinflammatory cytokines. However, whether these effects are direct or indirect is unclear.

In conclusion, the present results indicated that VILI depends on the activation of the calcineurin/NFATc4 pathway, which then leads to increases in the release of adhesion molecules and proinflammatory cytokines responsible for inducing the activation of inflammation in the lungs. Since the present study assessing the mechanism of VILI mainly focused on the calcineurin/NFATc4 pathway, the role of other members of the NFAT family has not been established. Therefore, other mechanisms may be involved in VILI.

\section{Acknowledgements}

Not applicable.

\section{Funding}

The present study were supported by The National Natural Science Fund (grant no. 81401626) and Research projects of Subei People's Hospital (grant no. yzucms201714).

\section{Availability of data and materials}

The datasets used and/or analyzed during the current study are available from the corresponding author on reasonable request.

\section{Authors' contributions}

DS designed the study and the experiments. ML and XF performed the majority of the experiments. ML wrote the manuscript. XF and XL analyzed the data. YZ, YX, XM and $\mathrm{XY}$ helped with experiments, especially in vitro cell cultures and western blot analysis.

\section{Ethics approval and consent to participate}

All experimental operations were performed with approval by and in accordance with the guidelines set by the Animal Ethics Committee of YangZhou University.

\section{Patient consent for publication}

Not applicable.

\section{Competing interests}

The authors declare that they have no competing interests.

\section{References}

1. Slutsky AS and Ranieri VM: Ventilator-induced lung injury. N Engl J Med 369: 2126-2136, 2013.

2. Cressoni M, Gotti M, Chiurazzi C, Massari D, Algieri I, Amini M, Cammaroto A, Brioni M, Montaruli C, Nikolla K, et al: Mechanical power and development of ventilator-induced lung injury. Anesthesiology 124: 1100-1108, 2016.

3. Yildiz C, Palaniyar N, Otulakowski G, Khan MA, Post M, Kuebler WM, Tanswell K, Belcastro R, Masood A, Engelberts D and Kavanagh BP: Mechanical ventilation induces neutrophil extracellular trap formation. Anesthesiology 122: 864-875, 2015.

4. Fang XZ, Huang TF, Wang CJ, Ge YL, Lin SY, Zhang Y and Gao J: Preconditioning of physiological cyclic stretch attenuated HMGB1 expression in pathologically mechanical stretch-activated A549 cells and ventilator-induced lung injury rats through inhibition of IL-6/STAT3/SOCS3. Int Immunopharmacol 31: 66-73, 2016.

5. Park SY, Kim HJ, Yoo KH, Park YB, Kim SW, Lee SJ, Kim EK, Kim JH, Kim YH, Moon JY, et al: The efficacy and safety of prone positioning in adults patients with acute respiratory distress syndrome: A meta-analysis of randomized controlled trials. J Thorac Dis 7: 356-367, 2015.

6. Wang T, Gross C, Desai AA, Zemskov E, Wu X, Garcia AN, Jacobson JR, Yuan JX, Garcia JG, Black SM, et al: Endothelial cell signaling and ventilator-induced lung injury: Molecular mechanisms, genomic analyses, and therapeutic targets. Am J Physiol Lung Cell Mol Physiol 312: L452-L476, 2017.

7. Tang T, Lang X, Xu C, Wang X, Gong T, Yang Y, Cui J, Bai L, Wang J, Jiang W and Zhou R: CLICs-dependent chloride efflux is an essential and proximal upstream event for NLRP3 inflammasome activation. Nat Commun 8: 202, 2017.

8. Schappe MS, Szteyn K, Stremska ME, Mendu SK, Downs TK, Seegren PV, Mahoney MA, Dixit S, Krupa JK, Stipes EJ, et al: Chanzyme TRPM7 mediates the $\mathrm{Ca}^{2+}$ influx essential for lipopolysaccharide-induced toll-like receptor 4 endocytosis and macrophage activation. Immunity 48: 59-74.e5, 2018.

9. Lu MC, Yu CL, Chen HC, Yu HC, Huang HB and Lai NS: Aberrant $\mathrm{T}$ cell expression of $\mathrm{Ca} 2+$ influx-regulated miRNAs in patients with systemic lupus erythematosus promotes lupus pathogenesis. Rheumatology 54: 343-348, 2015.

10. Guo Y, Yang X, He J, Liu J, Yang S and Dong H: Important roles of the $\mathrm{Ca}^{2+}$-sensing receptor in vascular health and disease. Life Sci 209: 217-227, 2018.

11. Michalick L, Erfinanda L, Weichelt U, van der Giet M, Liedtke W and Kuebler WM: Transient receptor potential vanilloid 4 and serum glucocorticoid-regulated kinase 1 are critical mediators of lung injury in overventilated mice in vivo. Anesthesiology 126: 300-311, 2017.

12. Pairet N, Mang S, Fois G, Keck M, Kühnbach M, Gindele J, Frick M, Dietl P and Lamb DJ: TRPV4 inhibition attenuates stretch-induced inflammatory cellular responses and lung barrier dysfunction during mechanical ventilation. PLoS One 13: e0196055, 2018.

13. Shibasaki F, Hallin $U$ and Uchino $H$ : Calcineurin as a multifunctional regulator. J Biochem 131: 1-15, 2002.

14. Li S, Pan Y, Ke R, Xie X, Zhai C, Shi W, Wang J, Yan X, Chai L, Wang Q, et al: Inhibition of phosphodiesterase-5 suppresses calcineurin/NFAT-mediated TRPC6 expression in pulmonary artery smooth muscle cells. Sci Rep 7: 6088, 2017. 
15. Yu BX, Yuan JN, Zhang FR, Liu YY, Zhang TT, Li K, Lv XF, Zhou JG, Huang LY, Shang JY and Liang SJ: Inhibition of Orail-mediated $\mathrm{Ca}^{2+}$ entry limits endothelial cell inflammation by suppressing calcineurin-NFATc 4 signaling pathway. Biochem Biophys Res Commun 495: 1864-1870, 2018.

16. Savage SR, Bretz CA and Penn JS: RNA-Seq reveals a role for NFAT-signaling in human retinal microvascular endothelial cells treated with TNFo. PLoS One 10: e0116941, 2015.

17. Karpurapu M, Lee YG, Qian Z, Wen J, Ballinger MN, Rusu L, Chung S, Deng J, Qian F, Reader BF, et al: Inhibition of nuclear factor of activated T cells (NFAT) c3 activation attenuates acute lung injury and pulmonary edema in murine models of sepsis. Oncotarget 9: 10606-10620, 2018.

18. Ranjan R, Deng J, Chung S, Lee YG, Park GY, Xiao L, Joo M, Christman JW and Karpurapu M: The transcription factor nuclear factor of activated $\mathrm{T}$ cells $\mathrm{c} 3$ modulates the function of macrophages in sepsis. J Innate Immun 6: 754-764, 2014.

19. Schmittgen TD and Livak KJ: Analyzing real-time PCR data by the comparative C(T) method. Nat Protoc 3: 1101-1108, 2008.

20. Li Q, Ge YL, Li M, Fang XZ, Yuan YP, Liang L and Huang SQ miR-127 contributes to ventilator-induced lung injury. Mol Med Rep 16: 4119-4126, 2017.

21. Zhang R, Pan Y, Fanelli V, Wu S, Luo AA, Islam D, Han B, Mao P, Ghazarian M, Zeng W, et al: Mechanical stress and the induction of lung fibrosis via the midkine signaling pathway. Am J Respir Crit Care Med 192: 315-323, 2015.

22. Iwaki $M$, Ito $S$, Morioka $M$, Iwata $S$, Numaguchi $Y$, Ishii $M$, Kondo M, Kume H, Naruse K, Sokabe M and Hasegawa Y: Mechanical stretch enhances IL-8 production in pulmonary microvascular endothelial cells. Biochem Biophys Res Commun 389: 531-536, 2009.
23. Lin JY, Jing R, Lin F, Ge WY, Dai HJ and Pan L: High tidal volume induces mitochondria damage and releases mitochondrial DNA to aggravate the ventilator-induced lung injury. Front Immunol 9: 1477, 2018.

24. Curley GF, Laffey JG, Zhang H and Slutsky AS: Biotrauma and ventilator induced lung injury: Clinical implications. Chest 150: 1109-1117, 2016.

25. Ni C, Li Z, Qian M, Zhou Y, Wang J and Guo X: Isoflurane induced cognitive impairment in aged rats through hippocampal calcineurin/NFAT signaling. Biochem Biophys Res Commun 460: 889-895, 2015

26. Miyakawa H, Woo SK, Dahl SC, Handler JS and Kwon HM: Tonicity-responsive enhancer binding protein, a rel-like protein that stimulates transcription in response to hypertonicity. Proc Natl Acad Sci USA 96: 2538-2542, 1999.

27. Imanaka H, Shimaoka M, Matsuura N, Nishimura M, Ohta N and Kiyono H: Ventilator-induced lung injury is associated with neutrophil infiltration, macrophage activation, and TGF-beta 1 mRNA upregulation in rat lungs. Anesth Analg 92: 428-436, 2001.

This work is licensed under a Creative Commons Attribution-NonCommercial-NoDerivatives 4.0 International (CC BY-NC-ND 4.0) License. 